Service social

\title{
L'approche communautaire en centre local de services communautaires : les enjeux en cause et les conditions requises
}

\section{Denis Bourque}

Volume 34, numéro 2-3, 1985

L'organisation communautaire

URI : https://id.erudit.org/iderudit/706276ar

DOI : https://doi.org/10.7202/706276ar

Aller au sommaire du numéro

Éditeur(s)

École de service social de l'Université Laval

ISSN

1708-1734 (numérique)

Découvrir la revue

Citer cet article

Bourque, D. (1985). L’approche communautaire en centre local de services communautaires : les enjeux en cause et les conditions requises. Service social, 34(2-3), 328-339. https://doi.org/10.7202/706276ar d'utilisation que vous pouvez consulter en ligne. 


\title{
L'approche communautaire en centre local de services communautaires : les enjeux en cause et les conditions requises *
}

\author{
Denis Bourque
}

Depuis quelque temps, le concept d'approche communautaire, ou encore d'approche des services socio-communautaires, est mis de l'avant dans le discours et les documents du Ministère des affaires sociales (M.A.S.) et de la Fédération des centres locaux de services communautaires (F.C.L.S.C.). Or, la publication récente du document ministériel intitulé "Le partage des responsabilités C.S.S.-C.L.S.C. en matière de services sociaux" ${ }^{1}$ est venue préciser, et en quelque sorte matérialiser ce modèle d'approche communautaire.

Afin de bien saisir le sens et la portée de ce concept, nous analyserons la situation qui en suscita l'élaboration, ses origines historiques selon le M.A.S. et la F.C.L.S.C. et nous en étudierons la définition, le contenu et les caractéristiques. Nous dégagerons ensuite ce qui apparaît être les enjeux sous-jacents à ce concept au moyen d'un essai d'analyse critique de ce nouveau discours communautarisant. Finalement, nous poserons un certain nombre de conditions essentielles à une approche véritablement communautaire.

Au point de départ, il importe de cerner ce que recoupe la notion d'approche communautaire. II s'agit essentiellement d'une stratégie d'intervention qui mise sur le potentiel des individus, des réseaux primaires, des groupes, des ressources communautaires et alternatives ainsi que sur les collectivités en général en ce qui a trait à la solution des problèmes sociaux et de santé, dans une perspective de remise en question de la place et du rôle des services publics et de l'ÉtatProvidence. 


\section{Les éléments déclencheurs dans la conjoncture}

"Aujourd'hui, cependant, cet État-Providence est presque partout en difficulté : le ralentissement de la croissance économique provoque un ralentissement équivalent de la croissance des ressources de l'État ; de ce simple fait, les coûts de la protection sociale et des services collectifs apparaissent de plus en plus lourds à supporter économiquement; par ailleurs, la diminution des activités économiques entraîne, d'elle-même, des conséquences sociales qui se traduisent, en retour, en une demande supplémentaire placée sur l'État, alors même que celui-ci est confronté à un plafonnement de ses ressources. ${ }^{2}$

La période actuelle se caractérise en effet par l'ampleur de la crise économique et de ses conséquences en termes de détérioration des conditions de vie d'une grande partie de la population. De plus, la crise économique a également causé une certaine diminution des revenus de l'État alors que, par ailleurs, les choix politiques des gouvernements ont engagé une proportion toujours croissante de ces revenus au soutien de l'économie. Ainsi, pour être en mesure de poursuivre cette politique, l'État doit limiter ses autres dépenses principalement effectuées dans les domaines de l'éducation, de la santé et des services sociaux.

Or, le M.A.S. porte un jugement très sévère sur l'efficience actuelle des services sociaux au Québec.

"Ils tendent [les services sociaux] à se traduire par une duplication et une mauvaise utilisation des ressources disponibles, une sur-utilisation des ressources les plus lourdes et les plus coûteuses en plus de limiter le développement des pratiques axées sur la prévention et l'utilisation de ressources légères, non institutionnelles et communautaires. ${ }^{3}$

La sous-ministre adjointe à la Direction générale des programmes de services sociaux, Madame Jeanne-d'Arc Vaillant, va encore plus loin en affirmant :

"Je vous dirai sans ménagement que la pratique sociale au Québec me paraît particulièrement mal en point [...] et les travailleurs sociaux doivent réfléchir et remettre en question les interventions et les approches afin de permettre la moins mauvaise adéquation possible de nos institutions et de nos pratiques aux besoins à satisfaire qui sont plus nombreux. $»^{4}$

La conjoncture à l'origine du concept de l'approche communautaire peut se résumer ainsi: la crise économique a provoqué une remise en question de l'État-Providence en même temps qu'elle a augmenté les demandes et les besoins des utilisateurs et utilisatrices 
des programmes et des services sociaux. Le défi de l'État est de réussir à faire plus avec moins de ressources.

\section{Les origines historiques du concept}

Pour le M.A.S. et la F.C.L.S.C., il faut trouver les origines du concept des services sociaux communautaires dans les travaux de la Commission Castonguay-Nepveu et dans la Loi 65.

"La Commission Castonguay-Nepveu aura particulièrement insisté pour que le système de distribution des services sociaux au Québec soit substantiellement réformé de manière à permettre la mise en place de services de plus en plus diversifiés, axés sur la prévention, rendus près des milieux de vie des citoyens, de façon à empêcher qu'en l'absence de ressources légères, des personnes ne soient contraintes d'utiliser des ressources plus lourdes. $n^{4}$

De plus, pour le M.A.S., la Commission Castonguay-Nepveu avait proposé les voies nouvelles qu'aurait dû, depuis, emprunter la pratique sociale. Ces voies nouvelles s'articulaient autour des notions de déprofessionnalisation, responsabilisation du citoyen face à sa situation, prise en charge individuelle et collective, prévention et solidarité communautaire. ${ }^{5}$

Nous pouvons nous interroger sur le lien réel entre l'orientation donnée à l'État-Providence par les travaux de la Commission, à cette époque, et l'actuel effort de redéfinition de la pratique sociale autour du concept de l'approche communautaire. Cette référence à l'esprit et à la lettre de la Loi 65 a ceci de commode : pouvoir présenter le projet des transferts C.S.S.-C.L.S.C. comme une mesure administrative allant de soi, en évitant ainsi le recours à de nouvelles législations ou réglementations.

Or, à la Fédération des C.L.S.C., on pense un peu différemment. "Le M.A.S. s'apprête à procéder à un nouveau partage des responsabilités entre les C.S.S. et les C.L.S.C. II s'agit là, à plus d'un titre, d'une décision majeure. Fondamentalement, elle rend possible le renouvellement des pratiques psycho-sociales au Québec. " ${ }^{6}$

Dans l'optique d'une réforme majeure des programmes et services sociaux, le concept à l'étude trouvera peut-être ses origines dans un passé plus récent. Ainsi le Rapport Barclay ${ }^{7}$ sur l'organisation des services sociaux et la pratique du service social en Angleterre pourrait avoir joué un rôle important à ce chapitre. On y décrit l'évolution des politiques sociales, en Grande-Bretagne, selon trois approches qui se sont successivement développées : celle de "filet de recours"; celle de 
l'État-Providence; et "l'approche communautaire", présentement en gestation.

Cette dernière postule que les individus ont davantage de potentiel, d'habileté et d'intérêt pour s'entraider et se supporter que ne le croyait l'approche de l'État-Providence. Les politiques d'intervention visent alors à équiper et supporter les réseaux informels, lorsqu'ils existent, à les créer ou à les développer lorsqu'ils sont inexistants ou trop faibles. La communauté y est donc perçue comme étant le principal fournisseur d'aide et de support aux individus et aux groupes. ${ }^{8}$

De plus, selon le Rapport Barclay, ce qui doit être remis en cause, ce sont le rôle et le mode de fonctionnement des services sociaux, les relations qu'ils entretiennent avec la communauté et leurs usagers et ainsi, fondamentalement, le rôle de l'État.

Il semblerait donc y avoir au moins, sinon plus d'inspiration dans ce Rapport que dans celui de la Commission Castonguay-Nepveu en ce qui a trait à la conceptualisation et à l'opérationnalisation de l'approche communautaire.

\section{Objectifs et caractéristiques de l'approche communautaire}

Selon le M.A.S., l'approche communautaire viserait ultimement l'objectif suivant :

"En reconnaissant la primauté de la responsabilité des citoyens, des réseaux primaires et communautaires, face au développement social des milieux, en même temps que la nécessité de développer, de supporter et de privilégier les services d'assistance de type communautaire dans la solution à apporter aux problèmes sociaux des personnes et des communautés, le présent cadre de référence rappelle l'un des principaux objectifs de la réforme socio-sanitaire, celui de favoriser la participation des citoyens à l'amélioration de leur état de santé et à l'adaptation de leur fonctionnement individuel et social. „9

Cet objectif pourra être poursuivi grâce à un renouvellement de la pratique sociale qui devrait désormais mettre l'accent sur le regroupement de personnes ayant des problèmes communs, de manière à permettre aux individus de sortir de leur isolement, en leur offrant tout à la fois l'aide personnalisée requise et l'opportunité de s'impliquer avec d'autres dans l'action sociale. Ainsi, les individus et les communautés seraient plus autonomes et plus aptes à se réaliser, davantage conscients et donc plus compétents pour agir sur leur situation.

La stratégie d'intervention consistera à mettre davantage à contribution les ressources de la communauté et à aider à faire renaître des 
solidarités, des attitudes d'entraide (bénévolat) et de participation qui ne sont plus suffisamment présentes dans la communauté.

De façon plus pratique, le M.A.S. entrevoit un rôle important pour les ressources des réseaux primaires et communautaires issues ou soutenues par l'approche communautaire.

"Lorsque ces ressources seront impuissantes à répondre aux besoins exprimés, le réseau public de services socio-communautaires devra être mis à contribution [...] et ceux-ci ne seront justifiés d'intervenir que lorsque la perte, la diminution ou l'absence d'autonomie sera associée à une carence temporaire ou définitive, partielle ou totale des réseaux primaires ou communautaires. ${ }^{10}$

Dans cette perspective, l'approche communautaire se caractérise, au niveau de la pratique, par les trois éléments suivants :

1. Utilisation de techniques d'intervention permettant de rejoindre plus efficacement plus de gens à moindre coût, tout en favorisant l'acquisition de la plus grande autonomie possible par les usagers (campagnes d'éducation, groupe d'entraide, intervention de réseaux, stimulation de l'entraide, etc.).

2. Développement du bénévolat, organisé dans une perspective de complémentarité par rapport aux services existants.

3. Mise en place et développement de ressources autonomes offrant des services sociaux communautaires susceptibles de répondre aux nouvelles demandes du milieu.

Pour la F.C.L.S.C., l'approche communautaire interpelle la réalité sociale actuelle selon trois niveaux : celui de l'État; celui de l'organisation des services; celui des pratiques sociales elles-mêmes. ${ }^{11}$

En ce qui a trait au rôle de l'État, il faudrait modifier les orientations en fonction desquelles il intervient et revoir le rôle du citoyen selon des perspectives de solidarité et de responsabilisation. En s'inspirant de Rosanvallon, ${ }^{12}$ la F.C.L.S.C. met de l'avant, comme éléments centraux d'une redéfinition de l'État-Providence: une plus grande visibilité sociale entre les prélèvements fiscaux individuels et leur utilisation collective; une réduction de la demande à l'État par un transfert de cette demande vers les collectivités locales et des ressources non publiques; le développement et l'utilisation des solidarités de base.

Quant au niveau de l'organisation des services, le Rapport Barclay apparaît être une forme d'opérationnalisation, pour les services sociaux, des orientations présentées plus tôt en regard de l'État. Selon ce Rapport, il est impossible que les services sociaux soient véritablement communautaires sans être structurellement intégrés aux communautés 
locales elles-mêmes. Ainsi, le rôle et le mode de fonctionnement des services sociaux, les politiques d'intervention ainsi que les relations entretenues avec les communautés locales et les clientèles doivent être remises en cause.

Enfin, le niveau des pratiques elles-mêmes doit reposer prioritairement sur les notions de réseaux de support naturel, d'entraide, d'aide et d'aidants naturels, de bénévolat et de pratiques de réseau. L'intérêt pour les systèmes de support naturel rejoint le désir d'établir, pour la prise en charge des problèmes, un nouveau partage des responsabilités entre l'État et les ressources communautaires locales non publiques ainsi qu'entre les intervenants et les membres des milieux naturels. La F.C.L.S.C. conclut sur cette question en affirmant un postulat considéré fondamental : toute modification dans la nature et les orientations de la pratique sociale actuelle ne pourra se réaliser sans s'insérer dans un renouvellement des perspectives par rapport au mode d'organisation des services et par rapport à l'État lui-même. ${ }^{13}$

\section{Analyse critique du concept d'approche communautaire}

Pour le M.A.S. et la F.C.L.S.C., l'approche communautaire est avant tout la résultante, au niveau des pratiques d'intervention, d'une redéfinition du rôle de l'État et de l'organisation des services sociosanitaires publics.

Cette redéfinition et cette réorganisation sont elles-mêmes la réponse à la situation suivante: d'une part, la demande de services de toute sorte s'accroîtra au cours des prochaines années en raison de l'augmentation des besoins liés au vieillissement de la population, à la marginalisation sociale des jeunes et à la nécessité de compenser les conséquences d'un taux élevé et chronique de chômage; d'autre part, la relance économique devant être faible, la capacité de payer de l'État continuera à être restreinte alors que la priorité accordée au soutien de l'économie drainera vers elle la plus grande partie des nouvelles ressources disponibles de l'État.

Dans ce contexte, les services publics sont jugés très contraignants et peu efficaces en raison de leurs coûts et d'un certain manque de souplesse causés par les exigences des conventions collectives et I'hyper-bureaucratisation des structures. L'heure est donc à la rationalisation accrue des services existants mais surtout à l'innovation et à la créativité :

" [...] afin de trouver des réponses encore plus efficaces, plus efficientes, plus souples ou moins onéreuses [...] Des concepts comme le développement des ressources du milieu ou la "prise en charge" 
pourraient acquérir dans ce contexte une signification encore plus fondamentale. " 14

C'est alors que sont revalorisées certaines notions connues sur les capacités naturelles des individus et des collectivités à solutionner leurs difficultés; sur la nécessité de favoriser l'autonomie et le contrôle des usagers face à leur devenir et, finalement, sur la nécessaire déprofessionnalisation et débureaucratisation des interventions des services publics.

\section{Quelques enjeux sous-jacents}

L'approche communautaire, telle que véhiculée par le M.A.S. et la F.C.L.S.C., comporte des éléments intéressants et prometteurs.

Ainsi, l'objectif ultime doit effectivement être la promotion et l'optimisation du développement individuel et collectif au moyen d'une prise de conscience et d'une prise en charge de leur situation et des solutions à leur portée par des individus et des groupes.

Par ailleurs, face à l'accroissement des problèmes sociaux, il faut en effet réorienter les structures et la pratique vers le champ de la prévention et de l'intervention collective et aussi favoriser le développement et l'utilisation maximale des ressources de la communauté.

Finalement, il importe de reconnaître le principe de primauté des réseaux primaires et secondaires et celui de la responsabilisation des citoyens et des groupes par rapport aux problèmes sociaux. II faut concevoir, de ce fait, l'intervention professionnelle comme une relation d'aide et de soutien envers l'individu et le groupe afin de les rendre plus autonomes et plus aptes à se réaliser, davantage conscients et plus compétents pour agir sur leur environnement.

Cependant, certaines interrogations se posent. Premièrement, la préoccupation omniprésente de réduire les coûts en matière de services sociaux ne risque-t-elle pas de subordonner et d'hypothéquer l'objectif d'améliorer la quantité et la qualité des services, surtout en période d'augmentation des besoins?

Deuxièmement, le concept de partenariat entre le réseau des Affaires sociales et les organismes communautaires autonomes ne risque-t-il pas de glisser vers la consolidation d'un système privé de première ligne dans le domaine social et sanitaire? Et, si oui, ne risquet-on pas de revenir à la notion de "charité privée" des années 1950 avec son cortège d'inégalités, de disparités régionales et d'arbitraire?

Troisièmement, la prise en charge individuelle et familiale de certaines problématiques socio-sanitaires et le développement du 
bénévolat de services ne seront-ils pas forcément le lot presque exclusif des femmes, dans notre société ? Par ailleurs, si la réduction des coûts souhaitée se traduisait en termes de chute de l'emploi dans les services publics, serait-ce encore une fois les femmes qui en subiraient principalement les conséquences?

Finalement, une vision étroite et technocratique de l'approche communautaire ne favoriserait-elle pas un processus de déresponsabilisation de l'État face aux problèmes sociaux et une remise en question de l'universalité, de l'accessibilité et de la gratuité de ces services?

Cette dernière question mérite qu'on s'y arrête car c'est le Ministre des affaires sociales de l'époque, Monsieur Pierre-Marc Johnson, qui déclarait, à la clôture du Congrès de la F.C.L.S.C. d'octobre 1983: "Les services de santé, ça c'est garanti. Quant aux services sociaux, cela est d'une autre nature. II faut émonder dans les programmes pour desservir les personnes âgées [...] On ne peut pas répondre à tous les besoins. " 15 Encore une fois, la F.C.L.S.C. apporte un éclairage encore plus lumineux en écrivant :

"Car on peut se demander si ce qui commence à être remis en cause actuellement et le sera probablement de plus en plus, ce n'est pas finalement le principe de l'universalité de l'accès à une partie des services qui, jusqu'ici, ont été assurés par les organismes publics. „ 16

Or, si la finalité de l'approche communautaire se résume à faciliter le désengagement de l'État en matière de services actuellement à sa charge, il semble alors que nous passerions à côté des véritables enjeux portés depuis longtemps par l'intervention communautaire.

En effet, bien avant la remise en cause de l'État-Providence se posait déjà un certain nombre de problèmes de fond:

1. Quelles conséquences a l'intervention de l'État, des institutions et des professionnels auprès des utilisateurs (individus et groupes) des services publics : contrôle, domination, intégration, dépendance?

2. Comment optimiser l'impact des services socio-sanitaires, l'approche globale et multidisciplinaire, le secteur de la prévention, le rôle de l'organisation communautaire et comment contrer la bureaucratie institutionnelle?

3. Quelle est la limite du modèle médical et thérapeutique, dans l'intervention psychosociale, par rapport aux modèles développemental, écologique ou structurel? 
4. Quelles sont les exigences d'une réelle participation démocratique et d'une prise en charge individuelle et collective favorisant les transformations des rapports avec l'environnement physique et social dans le sens d'une plus grande maîtrise des conditions de vie?

Ces questions, et bien d'autres, font l'objet d'une réflexion et d'une recherche continues depuis plusieurs années et ne peuvent trouver de réponses à l'intérieur de ce qui nous a été donné de lire au sujet de l'approche communautaire actuellement véhiculée par le M.A.S. et la F.C.L.S.C. Cependant, le contexte actuel de remise en question de l'État-Providence, de l'organisation des services sociosanitaires et de la pratique psychosociale, favorise l'émergence d'une conception de l'approche communautaire plus pertinente au questionnement présenté précédemment.

Sans prétendre posś́der les réponses aux questions fondamentales qui hantent les intervenantes et les intervenants depuis longtemps, nous aborderons maintenant ce qui ressort assez clairement comme des conditions essentielles pour que l'approche communautaire puisse jouer un rôle significatif dans un processus de promotion et d'optimisation du développement individuel et collectif.

\section{Quelques conditions pour une approche véritablement communautaire}

En premier lieu, il est primordial que l'approche communautaire vise fondamentalement l'objectif d'améliorer les conditions et la qualité de vie des individus, groupes et collectivités. Si l'expérimentation démontrait que cet objectif ne saurait être atteint par cette approche, il faudrait la remettre en question nonobstant l'éventuelle réduction des coûts qu'elle aurait pu entraîner.

En second lieu, l'État doit soutenir concrètement et suffisamment les réseaux primaires, les réseaux secondaires informels et formels, les ressources communautaires et alternatives ainsi que les groupes d'action communautaire et ce en termes de support professionnel, financier, technique et matériel. Ceci afin d'assurer que le milieu ne recevra pas que la responsabilité de la prise en charge de certains problèmes sociaux mais aussi les moyens et les ressources requis pour leur faire face. Par ailleurs, l'aide financière ne devrait pas servir de moyen de contrôle et d'intégration, subtil mais efficace, des organisations communautaires par l'État. Ce soutien concret et suffisant de l'État doit être disponible pour l'ensemble des problématiques sociales 
et de santé. Il ne saurait être question de confiner ce soutien, en raison de son évidente rentabilité, aux stricts domaines du maintien à domicile et de la désinstitutionnalisation. Par conséquent, les autres champs d'action collective et de prise en charge communautaire doivent pouvoir compter sur les mêmes ressources (logement, environnement, droits sociaux, consommation, santé, droits des femmes, etc.).

Troisièmement, les organismes, ressources et services issus de l'approche communautaire, ne doivent pas constituer une sorte de système privé parallèle de seconde zone où les principes d'universalité et de gratuité n'existeraient plus, et grâce auxquels le réseau public serait relégué à un rôle supplétif axé uniquement vers les personnes les plus démunies. Pour éviter cette situation, les principes d'universalité et de gratuité doivent être respectés au maximum. De plus, la ligne de démarcation entre l'intervention des ressources du milieu et celle des services publics devra se définir en fonction de la nature spécifique d'un besoin ou d'une problématique, et en fonction des capacités et compétences relatives de chacun de ces deux niveaux d'intervention en ce qui a trait à l'action pertinente à mener.

Il faudra également favoriser et assurer une véritable participation des bénéficiaires dans la définition, l'orientation et le contrôle des ressources et des services communautaires. Cette participation devra s'orchestrer avec celle des bénévoles et des salariés des mêmes structures, qui devront dépasser largement le rôle de simples exécutants pour prendre en main, démocratiquement, leurs organisations et y vivre une véritable expérience d'action collective.

En quatrième lieu, l'approche communautaire exige également un sérieux renouvellement des pratiques sociales dans le réseau public et aussi, en bonne partie, dans le réseau alternatif. Cette redéfinition devra être principalement l'œuvre des personnes qui distribuent les services, en collaboration avec celles qui les utilisent, individuellement ou en groupe ; et ceci dans une perspective nouvelle d'alliance plutôt que l'actuelle relation pouvoir/dépendance. Ceci mettra en cause la conception même des problèmes sociaux analysés, davantage en termes d'interactions entre différents facteurs environnementaux et l'individu ou le groupe plutôt qu'en termes de carences ou d'incapacités individuelles. II remettra également en question la conception de l'intervention professionnelle vécue, non plus comme la prise en charge du problème de l'usager par l'intervenant, mais bien réalisée, au travers de la transmission des connaissances, techniques et interrogations de l'intervenant, dans une démarche conjointe et consciente, vers la recherche d'une solution à une situation-problème. 
De plus, ce renouvellement devra favoriser une complémentarité entre les approches individuelles et collectives. Ceci en mettant l'accent sur une intervention, souvent simultanée, d'une part au niveau du développement de compétences individuelles permettant à l'individu de mieux composer avec son environnement et, d'autre part au niveau de la modification des facteurs environnementaux. Au chapitre du renouvellement des pratiques sociales, la formation appropriée des intervenants, à l'université et en cours d'emploi, devient une dimension centrale qui soulève sans doute elle-même ses propres conditions.

Enfin, l'organisation même des services sociaux et de santé devra permettre à l'approche communautaire de jouer pleinement son rôle. Ainsi, les activités et les programmes de prévention et d'intervention collective devront prendre une ampleur non équivoque et obtenir les ressources requises auprès des directions des établissements du réseau des Affaires sociales. De plus, ces mêmes directions devraient reconnaître les dangers posés par la bureaucratie, la centralisation et l'hyperhiérarchisation des structures, et développer une conception différente de la productivité et de l'évaluation en laissant plus de place à l'aspect qualitatif des interventions et à l'initiative des intervenants et des intervenantes.

Ces quelques conditions ne prétendent pas être exhaustives mais demeurent des éléments fondamentaux pour permettre à l'approche communautaire d'atteindre ses objectifs avoués. Il y aurait ainsi de meilleures garanties pour que celle-ci soit autre chose qu'un discours creux risquant, en bout de ligne, de servir de paravent à la déresponsabilisation sociale de l'État.

\section{Notes et références}

* Texte soumis en juillet 1985.

1 Ministère des affaires sociales (M.A.S.), Le partage des responsabilités C.S.S.C.L.S.C. en matière de services sociaux, Québec, 1984.

2 Ouellet, Hector, Les services sociaux communautaires: quelques éléments bibliographiques, Montréal, F.C.L.S.C., 1984. Texte présenté au Colloque des coordonnateurs des services communautaires des C.L.S.C. du Québec, Québec, le 11 avril 1984.

3 Ministère des affaires sociales, Cadre relatif au partage des responsabilités C.S.S.-C.L.S.C. en matière de services sociaux, Québec, 1983, p. 12.

4 VAILLANT, Jeanne-d'Arc, La pratique sociale de demain au Québec, allocution d'ouverture au congrès interrégional de la Corporation des travailleurs sociaux du Québec, Québec, le 21 octobre 1983, pp. 3 et 5.

${ }_{5}^{5}$ Ministère des affaires sociales, Le partage..., op. cit., pp. 6 et 7. 
6 Ouellet, H., op. cit., p. 1.

7 Le rapport Barclay est paru sous le titre: Social Workers, Their Roles and Tasks, publié pour le National Institute for Social Work, London, Bedford Square Press/Nevo, 1982.

${ }^{8}$ Ouellet, H., op. cit., pp. 8 et 9.

9 Ministère des affaires sociales, Le partage..., op. cit., p. 32.

10 Ibid., p. 28.

11 Ouellet, H., op. cit., p. 2.

12 Rosanvallon, Pierre, La crise de l'État-Providence, Paris, Seuil, 1981.

13 Ouellet, H., op. cit., pp. 25 et 26.

14 Fédération des centres locaux de services communautaires (F.C.L.S.C.), Les mutations de l'État-Providence et le devenir des C.L.S.C., Montréal, F.C.L.S.C., 1983, p. 47.

15 RowaN, René, "Johnson évoque la possibilité d'émonder dans les services sociaux", Le Devoir, 24 octobre 1983.

16 F.C.L.S.C., op. cit., p. 50. 